\title{
A Multidisciplinary Children's Airway Center: Impact on the Care of Patients With Tracheostomy
}

Kathleen A. Abode, BSN, MPH, a,b Amelia F. Drake, MD, b,c Carlton J. Zdanski, MD, b,c

George Z. Retsch-Bogart, MD, ${ }^{\mathrm{b}}$ Amanda B. Gee, BS, ${ }^{\mathrm{b}}$ Terry L. Noah, MD ${ }^{\mathrm{b}}$

BACKGROUND: Children with complex airway problems see multiple specialists. To improve outcomes and coordinate care, we developed a multidisciplinary Children's Airway Center. For children with tracheostomies, aspects of care targeted for improvement included optimizing initial hospital discharge, promoting effective communication between providers and caregivers, and avoiding tracheostomy complications.

METHODS: The population includes children up to 21 years old with tracheostomies. The airway center team includes providers from pediatric pulmonology, pediatric otolaryngology/head and neck surgery, and pediatric gastroenterology. Improvement initiatives included enhanced educational strategies, weekly care conferences, institutional consensus guidelines and care plans, personalized clinic schedules, and standardized intervals between airway examinations. A patient database allowed for tracking outcomes over time.

RESULTS: We initially identified 173 airway center patients including 123 with tracheostomies. The median number of new patients evaluated by the center team each year was 172. Median hospitalization after tracheostomy decreased from 37 days to 26 days for new tracheostomy patients $<1$ year old discharged from the hospital. A median of 24 care plans was evaluated at weekly conferences. Consensus protocol adherence increased likelihood of successful decannulation from $68 \%$ to $86 \%$ of attempts. The median interval of 8 months between airway examinations aligned with published recommendations.

conclusions: For children with tracheostomies, our Children's Airway Center met and sustained goals of optimizing hospitalization, promoting communication, and avoiding tracheostomy complications by initiating targeted improvements in a multidisciplinary team setting. A multidisciplinary approach to management of these patients can yield measurable improvements in important outcomes.

Children with complex airway problems, including those with tracheostomies, may receive care from multiple specialty providers. Achieving comprehensive, coordinated health care and accessing appropriate services present challenges. Diverse groups of providers may be managing comorbidities increasing the likelihood of disorganized care and poor communication. Special skills and education are required by health care providers and caregivers to competently care for these children., ${ }^{1,2}$ a University of North Carolina Health Care System, Chapel Hill, North Carolina; and Division of Pulmonology, Department of Pediatrics, and ${ }^{C}$ Division of Pediatric Otolaryngology, Department of Otolaryngology/Head and Neck Surgery, University of North Carolina School of Medicine, Chapel Hill, North Carolina

Ms Abode conceptualized and drafted the initial manuscript, developed clinical protocols and quality metrics, and carried out data collection and initial analysis; Drs Drake, Zdanski, and Retsch-Bogart developed clinical protocols and quality metrics, and reviewed and revised the manuscript; Ms Gee carried out data collection and reviewed and revised the manuscript; Dr Noah developed clinical protocols and quality metrics, carried out data analysis, and critically reviewed and revised the manuscript; and all authors approved the final manuscript as submitted.

\section{DOI: $10.1542 /$ peds.2015-0455}

Accepted for publication Jul 6, 2015

Address correspondence to Kathleen A. Abode, BSN, $\mathrm{MPH}$, Division of Pediatric Pulmonology, Department of Pediatrics, University of North Carolina-Chapel Hill, 450 MacNider, CB 7217, Chapel Hill, NC 27599. E-mail: jka@med.unc.edu

PEDIATRICS (ISSN Numbers: Print, 0031-4005; Online, 1098-4275).

Copyright (C) 2016 by the American Academy of Pediatrics

FINANCIAL DISCLOSURE: The authors have indicated they have no financial relationships relevant to this article to disclose.

FUNDING: The North Carolina Children's Airway Center project was supported by a grant from the Duke Endowment.

To cite: Abode KA, Drake AF, Zdanski CJ, et al A Multidisciplinary Children's Airway Center: Impact on the Care of Patients With Tracheostomy. Pediatrics. 2016;137(2):e20150455 
Numerous barriers to achieving comprehensive care were identified at our institution, a 150-bed tertiary care children's hospital including a 58-bed NICU and 20-bed PICU, with outpatient clinics throughout the facility. We recognized that for some children with new tracheostomies, hospital length of stay (LOS) was prolonged because of training and discharge planning delays. Outpatient subspecialty clinics used department-specific scheduling and communication systems. This led to inefficient, uncoordinated clinic scheduling resulting in patients making frequent trips to see providers from different services, and suboptimal communication compromising care for children with complex needs.

To address these problems and improve care, we developed a multidisciplinary Children's Airway Center in 2007, similar to those at a handful of major pediatric centers. ${ }^{3,4}$ For a subset of children with tracheostomies, our aim was to improve the following aspects of care: optimize LOS for children with newly placed tracheostomies; improve communication between providers and caregivers; and avoid complications of tracheostomy. Initiatives included an enhanced tracheostomy education program, fostering communication through weekly clinical care conferences, developing and implementing institutional consensus of care for aspects of care currently lacking published evidence-based or expert guidelines, and ensuring patients undergo periodic airway surveillance evaluation as recommended by the American Thoracic Society (ATS). ${ }^{5}$

\section{METHODS}

Patient Population, Personnel, and Infrastructure

Improvement initiatives were implemented and outcomes tracked via the center database at intervals from 2007 to 2013. Institutional review board approval was obtained for improvement initiatives. Inclusion criteria were children from birth to 21 years of age with congenital or acquired airway problems receiving care from multiple subspecialty services in the University of North Carolina Health Care system among pediatric pulmonology, pediatric otolaryngology/head and neck surgery, and pediatric gastroenterology (Fig 1). Children followed by subspecialists not actively participating in the center were excluded. Existing patients meeting criteria for inclusion comprised the initial group of airway center patients, with new patients added after discussion at weekly care conferences. Patients are actively followed by the center until the condition requiring multidisciplinary care is resolved or they transition to adult providers.

The center provides an organizational framework for efficient outpatient multidisciplinary care, existing as a "virtual clinic" rather than a stand-alone clinic. Patients move between preexisting hospital-based specialty clinics for same-day appointments with designated physicians, escorted by airway center staff if needed, while the center's nonphysician staff meets with patients and caregivers between physician appointments. Clinic visit schedules are tailored to individual patient and caregiver needs.

Administrative leadership of the center is shared by a pediatric pulmonologist and a pediatric otolaryngology/head and neck surgeon. A clinical program director (Ms Abode) oversees daily operations. An administrative coordinator manages a database of clinical data relevant to the center's care goals and is the initial contact for referrals and clinic appointments. A pediatric nurse practitioner(PNP) manages acute tracheostomy care and education. A respiratory

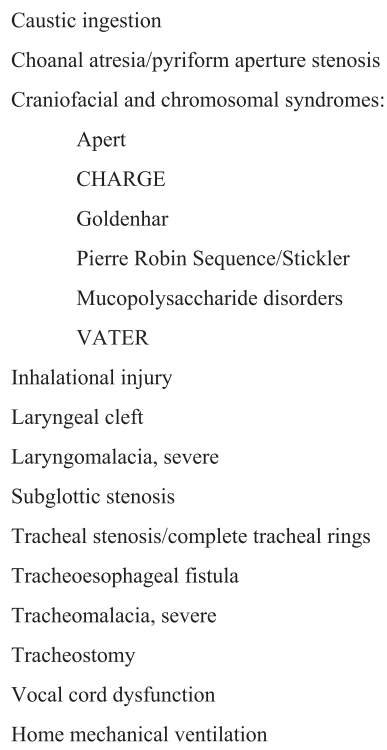

\section{FIGURE 1}

Selected airway related conditions of North Carolina's Children's Airway Center participants.

therapist (RT) coordinates home mechanical ventilation and manages the airway endoscopy video library. Partial support is provided for a social worker and a speech pathologist. Cross training of some functions of these individuals allows a relatively small staff to provide care in the absence of any one individual.

\section{Aim 1: Optimize LOS for Children With Newly Placed Tracheostomies}

Physician and nursing progress notes, case manager, social worker, and care conference notes were independently reviewed by the author (Ms Abode) and center PNP to characterize barriers to discharge associated with parental, health care, societal, and presence of disease factors previously described by others. ${ }^{6}$ We focused improvement efforts on enhancing staff and caregiver education and early initiation of caregiver training Educational tools were evaluated for content accuracy, gaps, and redundancies. The discharge coordination process was reviewed for timeliness and thoroughness, and new systems for education 
of providers and caregivers initiated. Education and training were informed by ATS guidelines. ${ }^{5}$ Process changes including checklists, revised educational strategies, and coordinated discharge planning were implemented over several years.

\section{Aim 2: Improve Communication Among Providers and Caregivers}

Weekly 1-hour care conferences to discuss patients' clinical status and active problems along with review of radiographic images, video, and still images of recent endoscopic examinations and surgical interventions serve as the primary setting for provider communication and collaboration. A weekly agenda, distributed before the conference, lists airway center patients requiring diagnostic or therapeutic decisionmaking. Current inpatients, those with upcoming or recent diagnostic or surgical procedures, new referrals, and patients requested by individual providers are included for focused discussion. Pediatric care providers from PICU, burn intensive care, anesthesiology, surgery, neurology/ sleep medicine, cardiology, and neonatology routinely attend airway center clinical care conferences for discussion of airway-related issues and specific information including identified barriers to care and test or treatment results. Physician and nonphysician provider input is encouraged.

\section{Aim 3: Avoid Complications of Tracheostomy}

Develop and Implement Institutional Consensus of Care for Decannulation

Core team meetings were held to examine current practice, review existing literature, and develop consensus of care for determining readiness for decannulation. . $^{5-13}$ The team reached consensus that tracheostomy decannulation should be preceded by airway endoscopy and followed by a 24to 48-hour hospital admission for observation. Patients decannulated in a location other than the operating room, bronchoscopy suite, or postanesthesia recovery room, decannulated without previous airway endoscopy, discharged $<24$ hours postdecannulation, or without a postdecannulation clinic visit within 6 weeks were considered to be outside the consensus guideline. Patients remaining decannulated for 6 months were defined as successfully decannulated. Patients failing an initial decannulation attempt, defined as inability to maintain baseline oxygen saturation and vital signs despite supplemental oxygen administration by nasal cannula or facemask, should be considered by their individual center provider for one or more interventions outlined in Fig 2.

\section{Ensure That Patients With \\ Tracheostomies Undergo Periodic Airway Surveillance Evaluation as Recommended in Published Guidelines}

Core team meetings were held to examine existing practices, review current literature and guidelines, ${ }^{5,14-17}$ and develop institutional consensus of care for frequency of airway surveillance evaluations. The process was informed by the 1999 ATS consensus document stating, "Children with chronic tracheostomies should undergo routine evaluation including rigid or flexible bronchoscopy every 6 to 12 months to assess the underlying airway pathology, detect and treat complications, assess tube size and position, and determine readiness for decannulation." ${ }^{5}$ For established tracheostomies, the group's consensus was that the maximum interval between airway examinations for children $\leq 30$ months of age should be 6 months, because of concern that rapid growth may necessitate frequent tracheostomy tube upsizing. The group agreed that children $>30$ months to 6 years should undergo airway examination annually, and children $>6$ years should be considered for airway examination every 1 to 2 years.

\section{Outcome Measures}

\section{Aim 1: Optimize LOS for Children With} Newly Placed Tracheostomies

LOS after new tracheostomy was calculated for children $<1$ year of age from 2007 to 2013. Patients $<1$ year of age are typically hospitalized at our institution before elective tracheostomy after unsuccessful extubation. This group was chosen for review as, in our experience, they tend to have similar indications for tracheostomy associated with pulmonary, craniofacial, or congenital disorders, or prematurity, ${ }^{7}$ and typically have similar discharge trajectories after tracheostomy.

Aim 2: Improve Communication Among Providers and Caregivers

The number of weekly clinical care conferences held per year and number of patients discussed per conference were tracked from 2007 to 2013.

\section{Aim 3: Avoid Complications of Tracheostomy}

To develop and implement institutional consensus of care for decannulation, all decannulation attempts documented in the medical record from 2007 to 2012 were reviewed to determine adherence to a consensus-driven decannulation algorithm, and characterized as successful or failed.

To ensure that patients with tracheostomies undergo periodic airway surveillance evaluation as recommended in published guidelines, patient age was recorded and intervals were measured between all airway evaluations documented in the medical record from 2008 to 2013.

\section{Analysis}

Descriptive statistics (median, interquartile range [IQR], range) 


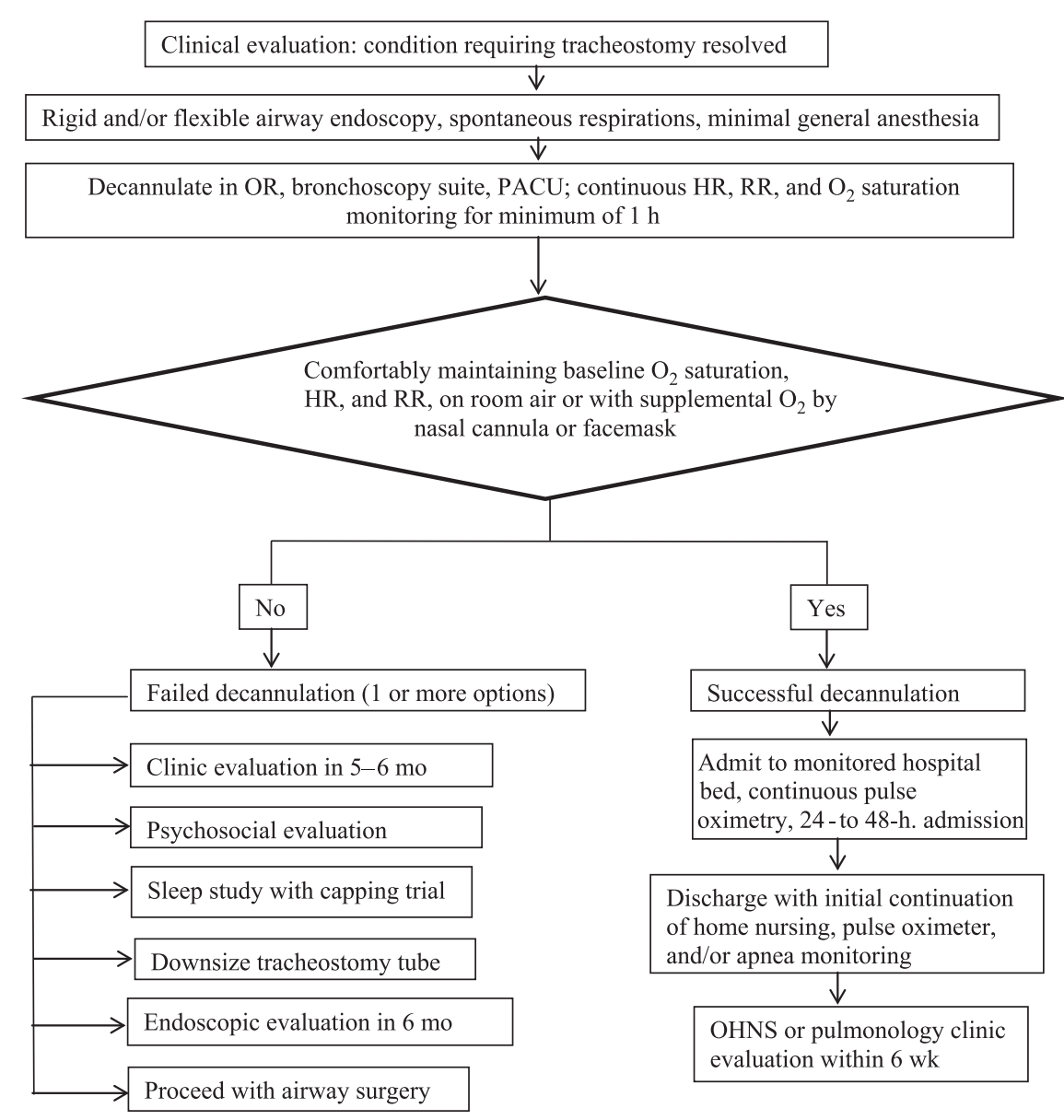

\section{FIGURE 2}

Consensus decannulation algorithm developed by the airway center. HR, heart rate; OHNS, otolaryngology/head and neck surgeon; OR, operating room; PACU, postanesthesia care unit; RR, respiratory rate.

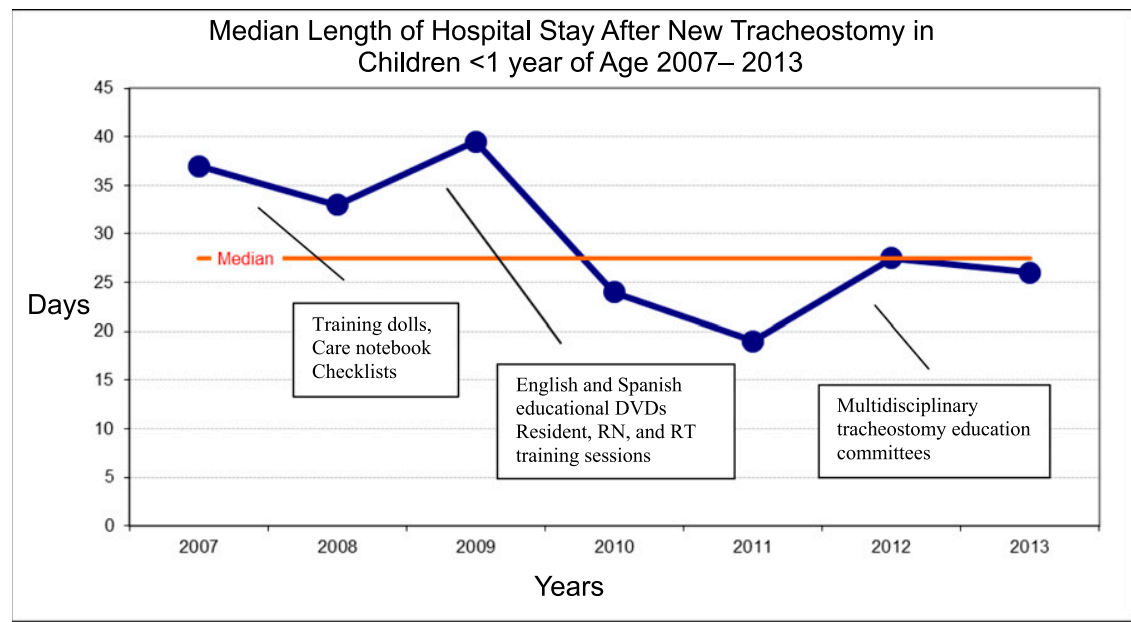

FIGURE 3

Median LOS in hospital after tracheostomy placement over time.

were used to report outcomes over time for all measures. A run chart describes interventions and progress in decreasing LOS after tracheostomy for children $<1$ year of age. The median line reflects the population median LOS of 27.5 days (IQR, 25-35; range, 19-39.5) from 2007 to 2013

(Fig 3).

\section{RESULTS}

Data for numbers of patients included in the airway center database including those with tracheostomies are shown in Fig 4. At the center's inception in 2007, there were 173 existing patients ages birth to 21 years with complex airway disorders at our institution who met previously stated criteria for inclusion in the airway center. Of these, 123 had existing tracheostomies. Between 2007 and 2013, there were 1273 patients evaluated by the center with a median of 172 new patients (IQR, 169-190; range, 144-317) added each year. The proportion of airway 
center patients with tracheostomies increased from $42 \%$ in 2007 to $64 \%$ in 2013.

\section{Aim 1: Optimize LOS for Children With Newly Placed Tracheostomies}

We identified the following barriers to timely discharge for children with new tracheostomies: delayed identification of caregivers requiring training; delayed initiation of caregiver education and training; lack of trained staff providing education; lack of appropriate and culturally diverse teaching tools; and delayed initiation of home nursing and supply requests. With a focus on enhancing educational strategies and early initiation of caregiver training, the center's PNP worked with inpatient nurses to update educational resources including written and audiovisual materials in English and Spanish and provide customized dolls for simulation. Teaching checklists ensured that caregiver training included all aspects of care. Staff training expanded to include physicians, registered nurses (RNs), and RTs to increase the number and availability of trainers. Modifications to discharge processes occurred simultaneously. Care management plans for patients being considered for elective tracheostomy are developed at weekly care

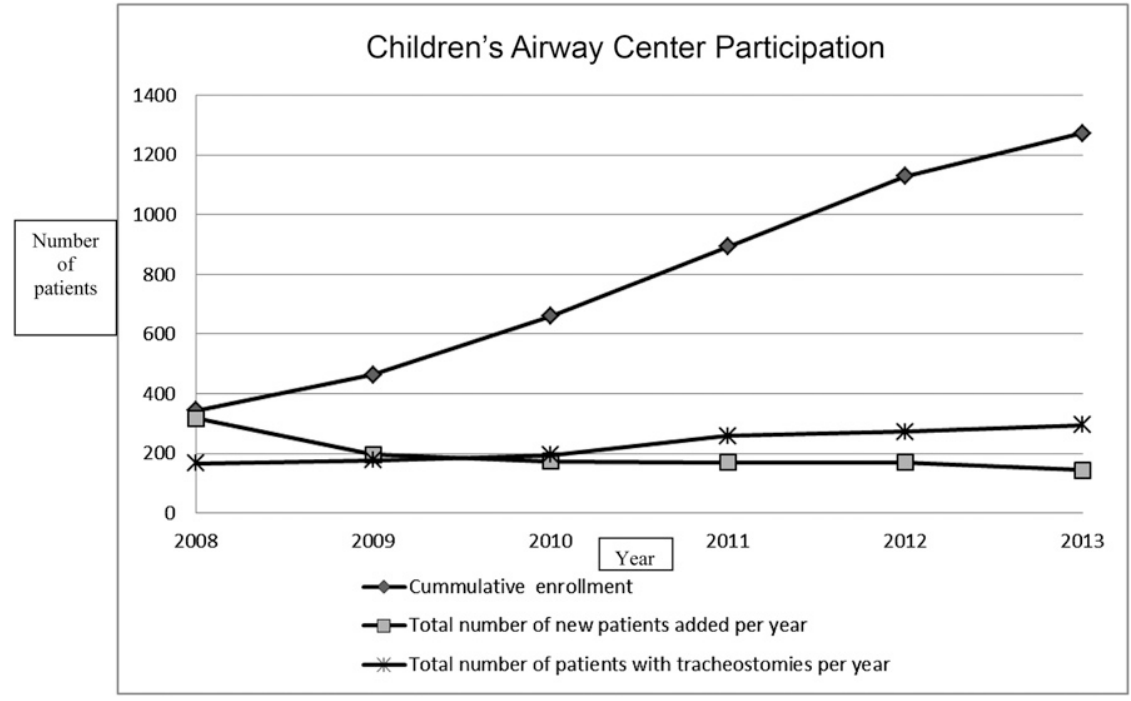

FIGURE 4

Number of patients included in the airway center program over time.

conferences and during caregiver centered discussions attended by the center's PNP and social worker. Initial teaching, community resource identification, and discharge planning are implemented when the decision is made to proceed with tracheostomy.

Improvements began in 2007. Demographics and changes in LOS over time are shown in Table 1 and Fig 3. In 2007, the median LOS after tracheostomy for children $<1$ year of age discharged to the caregiver's home was 37 days (IQR, 26.5-46.3; range, 22-83). LOS declined as improvements were implemented. In 2013, the mean LOS after tracheostomy for children $<1$ year of age discharged to the caregiver's home was 26 days (IQR, 15.8-38.5; range, 15-53).

\section{Aim 2: Improve Communication Among Providers and Caregivers}

The center's goal is weekly multidisciplinary meetings to ensure timely decision-making and action item follow-up. This format streamlines decision-making and avoids treatment delays associated

TABLE 1 Demographics of Children <1 Year of Age With New Tracheostomies 2007-2013

\begin{tabular}{|c|c|c|c|c|c|c|c|}
\hline Year & 2007 & 2008 & 2009 & 2010 & 2011 & 2012 & 2013 \\
\hline $\begin{array}{l}\text { Number of new tracheostomies } \\
\text { performed }\end{array}$ & 13 & 14 & 13 & 10 & 18 & 17 & 13 \\
\hline $\begin{array}{l}\text { Median (IQR; range) age at } \\
\text { tracheostomy in days }\end{array}$ & $\begin{array}{c}163(124-233.5 \\
19-326)\end{array}$ & $\begin{array}{c}38(24-55.5 \\
5-243)\end{array}$ & $\begin{array}{c}64.5(26-137.5 \\
5-188)\end{array}$ & $\begin{array}{c}175.5(57- \\
233.3 ; 9-348)\end{array}$ & $\begin{array}{c}87(70-214 \\
11-301)\end{array}$ & $\begin{array}{c}100 \text { (82.8-140; } \\
27-95)\end{array}$ & $\begin{array}{c}97(84.5-128.3 ; \\
28-239)\end{array}$ \\
\hline $\begin{array}{l}\text { Number of new tracheostomies } \\
\text { in infants born prematurely }\end{array}$ & 5 & 0 & 4 & 4 & 5 & 5 & 2 \\
\hline $\begin{array}{l}\text { Number of patients discharged } \\
\text { to caregiver home with } \\
\text { tracheostomy }\end{array}$ & 8 & 7 & 10 & 8 & 13 & 12 & 6 \\
\hline $\begin{array}{l}\text { Median (IQR; range) LOS in days } \\
\text { posttracheostomy }\end{array}$ & $\begin{array}{l}37 \text { (26.5-46.3; } \\
22-83)\end{array}$ & $\begin{array}{c}33(32-43 ; \\
27-95)\end{array}$ & $\begin{array}{c}39.5(28.5-58.8 \\
17-98)\end{array}$ & $\begin{array}{c}24(18-39.5 \\
18-106)\end{array}$ & $\begin{array}{c}19(13-44 ; \\
10-111)\end{array}$ & $\begin{array}{c}27.5(25.8-5.5 \\
11-81)\end{array}$ & $\begin{array}{c}26(15.8-38.5 \\
15-53)\end{array}$ \\
\hline $\begin{array}{l}\text { Number of patients discharged } \\
\text { on ventilator }\end{array}$ & 0 & 1 & 3 & 0 & 3 & 1 & 2 \\
\hline $\begin{array}{l}\text { Number of patients readmitted } \\
\text { within } 30 \mathrm{~d} \text { of initial discharge }\end{array}$ & 0 & 0 & 0 & 3 & 4 & 4 & 1 \\
\hline
\end{tabular}

a Patients who died, were decannulated before discharge, or were transferred to another institution are excluded

${ }^{b}$ Ten of 12 patients readmitted for respiratory infections, 1 patient admitted in 2011 for possible shunt infection, and 1 patient admitted in 2013 for additional caregiver training. 
with separate evaluations by multiple providers. The administrative coordinator sets the conference agenda: a brief summary of current health status, and specific issues requested for discussion. Succinct conversation primarily focuses on coordinating diagnostic and treatment plans. Additional time is devoted to review of select videos of airway pathology with discussion of medical and surgical treatment options. The clinical director moderates the conference and it is summarized for the electronic medical record, database entry, and e-mail distribution by the administrative coordinator. Attendees learn strategies for coordinating care for children with complex disorders, understand the care roles of each team member, and improve understanding of complex airway pathology and treatment modalities.

Conferences were held intermittently in 2007 as the center team secured meeting space and developed the conference format. There were 39 weekly conferences in 2008, with an increase to 47 conferences by 2013. The median number of patients included on the weekly discussion agenda was 24 (IQR, 19-30; range, 3-47).

\section{Aim 3: Avoid Complications of Tracheostomy}

Between 2007 and 2012, there were 154 decannulation attempts in 142 patients. The institutional guideline was used in $64 \%$ of decannulation attempts with successful decannulation occurring in $71 \%$ of initial attempts using the guideline, and ultimately $86 \%$ of overall attempts using the guideline. For some patients, the consensus guideline was not used. Of this group, 59\% were successful at the initial attempt, with $68 \%$ of attempts ultimately resulting in successful decannulation.
After implementation of the center surveillance protocols, children $\leq 30$ months of age underwent 234 airway evaluations from 2008 to 2013 , with a median interval of 6 months (IQR, 4-8; range, 2-10) between examinations. Children $>30$ months to 6 years underwent 165 examinations with a median interval of 8 months (IQR, 5-13; range, 1-40) between examinations. Children $>6$ years underwent 217 examinations with a median interval of 11 months (IQR, 6-15; range, 0.5-35) between airway examinations.

\section{DISCUSSION}

To better address the care needs of a complex patient population, we designed a Children's Airway Center tailored to our institutional resources and focused on providing coordinated, multidisciplinary care. We identified 3 specific improvement aims for a subset of children with tracheostomies. For aim 1 (optimizing LOS for children with newly placed tracheostomies), a key finding was decreased LOS from 37 days to 26 days for children discharged to the caregiver's home after new tracheostomy. Readmission rates within 30 days were low (12/64), and mostly related to respiratory infections. For aim 2 (improving communication among providers and caregivers), multidisciplinary clinical care conferences increased in frequency to nearly weekly and this process success was sustained. For aim 3 (avoiding complications of tracheostomy), a consensus-driven decannulation protocol resulted in successful decannulation in $71 \%$ of initial attempts and $86 \%$ of overall attempts, and consistent, timely tracheostomy surveillance was achieved.

For young children with newly placed tracheostomies, we found that by focusing on early discharge planning and improving educational resources, we decreased median LOS by 11 days, aligning our LOS with reports by others. ${ }^{14,18-21}$ The initiative to improve communication resulted in implementation of weekly clinical care conferences focused on treatment planning, with video review and discussion of airway pathology contributing an educational component. Efforts to avoid tracheostomy complications led to applying a consensus-driven algorithm to the decannulation process increasing the likelihood of successful decannulation by $18 \%$, and aligning institutional standards with nationally recognized recommendations for interval airway surveillance examinations of 6 to 12 months.

Although little has been published about multidisciplinary children's airway centers, much has been written about multidisciplinary teams (MDTs) in specialties such as adult oncology, urology, and craniofacial surgery. ${ }^{22-28}$ The team care concept focuses on ensuring that providers with specific expertise are involved with the patient and the team responsible for the patient's care. ${ }^{29}$ Although some specialties and institutions have found MDTs to be costly, inefficient, and lacking in evidence for a positive impact on patient outcomes, ${ }^{24,27,30}$ one study demonstrates decreased time to decannulation and LOS for adults with tracheostomies managed by an MDT. ${ }^{31}$ The authors concluded that MDTs allow for consensus decisionmaking, and avoid delays associated with multiple separate evaluations. ${ }^{31}$ Another study revealed improved diagnostic workup efficiency and adherence to therapy when evidencebased guidelines were applied by an MDT in treating children with osteomyelitis. $^{32}$

The American Cleft Palate Craniofacial Association and Cleft Palate Foundation has adopted organizational standards identifying characteristics of quality teams 
focusing on composition and function, provides organizational recognition of MDTs, and promotes a coordinated evaluation and treatment approach within the framework of the patient's overall needs. ${ }^{33}$

It remains difficult to measure costs and benefits of MDTs because of the broad definitions of multidisciplinary working, difficulty estimating associated costs, and variability in outcomes associated with effective MDTs. Although we have not attempted to estimate cost savings of our center, potential savings of our approach include creating a model not requiring construction of new clinic facilities, decreasing LOS, coordinating multiple necessary procedures under a single anesthesia event, and timely decannulations. Organizational, patient-centered, and team-based outcomes should all be considered when evaluating the effectiveness of multidisciplinary care. Organizational outcomes include reduced hospital time, improved access to care, and decreased unanticipated admissions. Patient-centered outcomes include enhanced satisfaction, acceptance of treatment, and improved health outcomes. Team outcomes include enhanced job satisfaction and wellbeing, and greater role clarity and quality of decision-making and implementation. . $^{30,31,34,35}$

As part of a clinical quality improvement project, conclusions from our data have inherent limitations. The lack of a clear definition of multidisciplinary care makes it difficult to compare our results against other MDTs. It is possible that factors besides the airway center program altered LOS posttracheostomy or success rates of decannulation attempts. Maintenance of improvements over time provides some evidence that changes resulted from our interventions. Finally, our data focused on organizational or team outcomes, but ultimately, patient-centered outcomes may be the most important markers of success. Although not the focus of this report, additional benefits of the airway center are opportunities for education, research, and scholarship. Educational goals established for weekly care conferences meet criteria for awarding continuing medical education credit and continuing nursing education credit for attendance and participation. From a research perspective, airway center team members have collaborated with researchers on a project to develop predictive models for treatment of upper airway obstruction. ${ }^{36-39}$ Studies focusing on inhalation injury in pediatric burn patients, ${ }^{40}$ airway management of complex patients, ${ }^{41,42}$ and the experience of the school-aged child with a tracheostomy ${ }^{43}$ were also supported by airway center data and resources.

Multidisciplinary children's airway centers address the needs of a complex patient population and comprise unique patients relying on multiple providers and extensive resources. The primary value of such programs may lie in enhancing communication and thoughtful decision-making among caregivers and the medical community dedicated to caring for these children. Because airway centers are inherently complex systems, it may be difficult to develop standardized performance measures and assess outcomes across patient, team, and organizational domains. Organizations developing multidisciplinary children's airway centers should adapt structure, processes, and goals to individual institutional strengths, barriers, and resources.

\section{ACKNOWLEDGMENTS}

We thank Cynthia Reilly, PNP, Mark Hall, RRT, and Bradley Vaughn, MD, for their contributions to the Center team.

\section{ABBREVIATIONS}

ATS: American Thoracic Society

CHARGE: Coloboma of the eye, Heart defects, Atresia of the choanae, Retardation of growth and/or development, Genital and/or urinary abnormalities, and Ear abnormalities and deafness

IQR: interquartile range

LOS: length of stay

MDT: multidisciplinary team

VATER: Vertebral anomalies, Anal atresia, Cardiac defects, Tracheoesophageal fistula and/or Esophageal atresia, Renal and Radial anomalies

POTENTIAL CONFLICT OF INTEREST: The authors have indicated they have no potential conflicts of interest to disclose.

\section{REFERENCES}

1. Liu C, Heffernan C, Saluja S, et al. Indications, hospital course, and complexity of patients undergoing tracheostomy at a tertiary care pediatric hospital.
Otolaryngol Head Neck Surg. 2014; 151(2):232-239

2. Berry JG, Graham DA, Graham RJ, et al. Predictors of clinical outcomes and hospital resource use of children after tracheotomy. Pediatrics. 2009;124(2): 563-572

3. Cincinnati Children's Hospital Medical Center Aerodigestive Center. Available 
at: www.cincinnatichildrens.org/ service/a/aerodigestice/default/. Accessed December 28, 2014

4. Children's Hospital of Pittsburgh of University of Pittsburgh Medical Center Aerodigestive Center. Available at: www.chp.edu/CHP/aerodigestivecenter. Accessed December 29, 2014

5. Sherman JM, Davis S, AlbamontePetrick S, et al. Care of the child with a chronic tracheostomy. This official statement of the American Thoracic Society was adopted by the ATS Board of Directors, July 1999. Am J Respir Crit Care Med. 2000;161(1):297-308

6. Cross D, Leonard BJ, Skay CL, Rheinberger MM. Extended hospitalization of medically stable children dependent on technology: a focus on mutable family factors. Issues Compr Pediatr Nurs. 1998;21 (2):63-84

7. Gray RF, Todd NW, Jacobs IN. Tracheostomy decannulation in children: approaches and techniques. Laryngoscope. 1998;108(1 pt 1):8-12

8. Leung R, Berkowitz RG. Decannulation and outcome following pediatric tracheostomy. Ann Otol Rhinol Laryngol. 2005;114(10):743-748

9. Merritt RM, Bent JP, Smith RJ. Suprastomal granulation tissue and pediatric tracheotomy decannulation. Laryngoscope. 1997;107 (7):868-871

10. Midwinter KI, Carrie S, Bull PD. Paediatric tracheostomy: Sheffield experience 1979-1999. J Laryngol Otol. 2002;116(7):532-535

11. Mukherjee B, Bais AS, Bajaj Y. Role of polysomnography in tracheostomy decannulation in the paediatric patient. J Laryngol Otol. 1999;113(5):442-445

12. Sorin A, McCarthy JG, Bernstein JM. Predicting decannulation outcomes after distraction osteogenesis for syndromic micrognathia. Laryngoscope. 2004;114(10):1815-1821

13. Waddell A, Appleford R, Dunning C, Papsin BC, Bailey CM. The Great Ormond Street protocol for ward decannulation of children with tracheostomy: increasing safety and decreasing cost. Int J Pediatr Otorhinolaryngol. 1997;39(2): 111-118
14. Carr MM, Poje CP, Kingston L, Kielma D, Heard C. Complications in pediatric tracheostomies. Laryngoscope. 2001;111(11 pt 1):1925-1928

15. Tom LWC, Miller L, Wetmore RF, Handler SD, Potsic WP. Endoscopic assessment in children with tracheotomies. Arch Otolaryngol Head Neck Surg. 1993;119(3):321-324

16. Parrilla C, Scarano E, Guidi ML, Galli J, Paludetti G. Current trends in paediatric tracheostomies. Int J Pediatr Otorhinolaryngol. 2007;71(10):1563-1567

17. Eber E, Oberwaldner B. Tracheostomy care in the hospital. Paediatr Respir Rev. 2006;7(3):175-184

18. Duncan BW, Howell LJ, deLorimier AA, Adzick NS, Harrison MR. Tracheostomy in children with emphasis on home care. J Pediatr Surg. 1992;27 (4):432-435

19. Graf JM, Montagnino BA, Hueckel $\mathrm{R}, \mathrm{McPherson} \mathrm{ML}$. Pediatric tracheostomies: a recent experience from one academic center. Pediatr Crit Care Med. 2008;9(1):96-100

20. United States Department of Health and Human Services. Agency for Healthcare Research and Quality. Healthcare cost and utilization project. Available at: http://hcupnet.ahrq.gov. Accessed December 29, 2014

21. Lewis CW, Carron JD, Perkins JA, Sie KC, Feudtner C. Tracheotomy in pediatric patients: a national perspective. Arch Otolaryngol Head Neck Surg. 2003;129(5):523-529

22. Kurpad R, Kim W, Rathmell WK, et al. A multidisciplinary approach to the management of urologic malignancies: does it influence diagnostic and treatment decisions? Urol Oncol. 2011;29(4):378-382

23. Wheless SA, McKinney KA, Zanation AM. A prospective study of the clinical impact of a multidisciplinary head and neck tumor board. Otolaryngol Head Neck Surg. 2010;143(5):650-654

24. De leso PB, Coward JI, Letsa I, et al. A study of the decision outcomes and financial costs of multidisciplinary team meetings (MDMs) in oncology. $\mathrm{Br}$ $J$ Cancer. 2013;109(9):2295-3000
25. Munro AJ, Swartzman S. What is a virtual multidisciplinary team (vMDT)? Br J Cancer. 2013;108(12):2433-2441

26. Gort M, Broekhuis M, Regts G. How teams use indicators for quality improvement: a multiple-case study on the use of multiple indicators in multidisciplinary breast cancer teams Soc Sci Med. 2013;96:69-77

27. Lamb BW, Brown KF, Nagpal K, Vincent C, Green JS, Sevdalis N. Quality of care management decisions by multidisciplinary cancer teams: a systematic review. Ann Surg Oncol. 2011;18(8):2116-2125

28. Bradley PJ. Multidisciplinary clinical approach to the management of head and neck cancer. Eur Arch Otorhinolaryngol. 2012;269(12):2451-2454

29. Spitzer R. Teamwork, teams, and reality. Nurse Lead. 2008;12:6

30. Bosch M, Faber MJ, Cruijsberg J, et al. Review article: Effectiveness of patient care teams and the role of clinical expertise and coordination: a literature review. Med Care Res Rev. 2009;66 (suppl 6):5S-35S

31. Tobin AE, Santamaria JD. An intensivistled tracheostomy review team is associated with shorter decannulation time and length of stay: a prospective cohort study. Crit Care. 2008;12(2):R48

32. Copley LA, Kinsler MA, Gheen T, Shar A, Sun D, Browne R. The impact of evidence-based clinical practice guidelines applied by a multidisciplinary team for the care of children with osteomyelitis. J Bone Joint Surg Am. 2013;95(8):686-693

33. American Cleft Palate-Craniofacial Association. Available at: www.acpacpf.org. Accessed December 28, 2014

34. Ke KM, Blazeby JM, Strong S, Carroll FE, Ness AR, Hollingworth W. Are multidisciplinary teams in secondary care cost-effective? A systematic review of the literature. Cost Eff Resour Alloc. 2013;11(1):7

35. Mickan SM. Evaluating the effectiveness of health care teams. Aust Health Rev. 2005;29(2):211-217

36. Calloway HE, Kimbell JS, Davis SD, et al. Comparison of endoscopic versus 3D CT derived airway measurements. Laryngoscope. 2013;123(9):2136-2141 
37. Davidson Ward SL, Amin R, Arens R, et al. Pediatric sleep-related breathing disorders: advances in imaging and computational modeling. IEEE Pulse. 2014;5(5):33-39

38. Hong Y, Davis B, Marron JS, et al. Statistical atlas construction via weighted functional boxplots. Med Image Anal. 2014;18(4):684-698

39. Wijesundara K, Zdanski C, Kimbell J, Price H, Iftimia N, Oldenburg AL.
Quantitative upper airway endoscopy with swept-source anatomical optical coherence tomography. Biomed Opt Express. 2014;5(3):788-799

40. Joyner BL, Jones SW, Cairns BA, et al. DNA and inflammatory mediators in bronchoalveolar lavage fluid from children with acute inhalational injuries. J Burn Care Res. 2013;34(3):326-333

41. Muhlebach MS, Shaffer CB, Georges L, Abode K, Muenzer J. Bronchoscopy and airway management in patients with mucopolysaccharidoses (MPS). Pediatr Pulmonol. 2013;48(6):601-607

42. Shadfar S, Drake AF, Vaughn BV, Zdanski CJ. Pediatric airway abnormalities: evaluation and management. Oral Maxillofac Surg Clin North Am. 2012;24(3):325-336

43. Patel MR, Zdanski CJ, Abode KA, et al. Experience of the school-aged child with tracheostomy. Int J Pediatr Otorhinolaryngol. 2009;73(7):975-980 


\section{A Multidisciplinary Children's Airway Center: Impact on the Care of Patients With Tracheostomy}

Kathleen A. Abode, Amelia F. Drake, Carlton J. Zdanski, George Z. Retsch-Bogart, Amanda B. Gee and Terry L. Noah

Pediatrics 2016;137;

DOI: 10.1542/peds.2015-0455 originally published online January 11, 2016;

\begin{tabular}{|ll}
$\begin{array}{l}\text { Updated Information \& } \\
\text { Services }\end{array}$ References & $\begin{array}{l}\text { including high resolution figures, can be found at: } \\
\text { http://pediatrics.aappublications.org/content/137/2/e20150455 }\end{array}$ \\
This article cites 39 articles, 2 of which you can access for free at: \\
http://pediatrics.aappublications.org/content/137/2/e20150455\#BIBL \\
This article, along with others on similar topics, appears in the \\
following collection(s): \\
Administration/Practice Management \\
http://www.aappublications.org/cgi/collection/administration:practice \\
management_sub
\end{tabular}




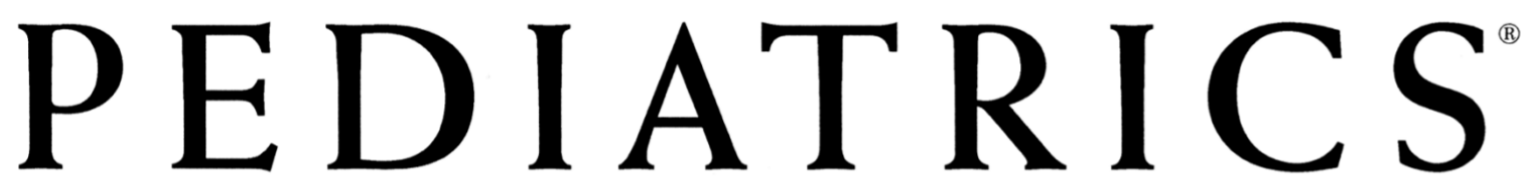

OFFICIAL JOURNAL OF THE AMERICAN ACADEMY OF PEDIATRICS

\section{A Multidisciplinary Children's Airway Center: Impact on the Care of Patients With Tracheostomy}

Kathleen A. Abode, Amelia F. Drake, Carlton J. Zdanski, George Z. Retsch-Bogart, Amanda B. Gee and Terry L. Noah

Pediatrics 2016;137;

DOI: 10.1542/peds.2015-0455 originally published online January 11, 2016;

The online version of this article, along with updated information and services, is located on the World Wide Web at: http://pediatrics.aappublications.org/content/137/2/e20150455

Pediatrics is the official journal of the American Academy of Pediatrics. A monthly publication, it has been published continuously since 1948. Pediatrics is owned, published, and trademarked by the American Academy of Pediatrics, 141 Northwest Point Boulevard, Elk Grove Village, Illinois, 60007. Copyright $\odot 2016$ by the American Academy of Pediatrics. All rights reserved. Print ISSN: 1073-0397. 